\title{
Increased expression of endocan in arthritic synovial tissues: Effects of adiponectin on the expression of endocan in fibroblast-like synoviocytes
}

\author{
KYOUNG SOO KIM ${ }^{1}$, YEON-AH LEE ${ }^{2}$, HYE-IN JI ${ }^{1}$, RAN SONG ${ }^{2}$, JUNG YEON KIM ${ }^{3}$, SANG-HOON LEE ${ }^{2}$, \\ SEUNG-JAE HONG ${ }^{2}$, MYUNG CHUL YOO ${ }^{1}$ and HYUNG-IN YANG ${ }^{2}$ \\ ${ }^{1}$ East-West Bone and Joint Research Institute, Kyung Hee University Hospital at Gangdong, Kyung Hee University; \\ ${ }^{2}$ Division of Rheumatology, Department of Internal Medicine, Kyung Hee University Hospital at Gangdong, \\ Kyung Hee University Medical School, Seoul 134-727; ${ }^{3}$ Department of Pathology, \\ Inje University Sanggye Paik Hospital, Seoul 139-707, Republic of Korea
}

Received March 19, 2014; Accepted August 14, 2014

DOI: $10.3892 / \mathrm{mmr} .2014 .3057$

\begin{abstract}
This study was performed to evaluate whether endocan expression, which is known to be involved in tumor angiogenesis, was increased in rheumatoid arthritic tissues. In addition, the involvement of adiponectin in the regulation of endocan expression in arthritic joints was examined. Arthritic synovial tissues from patients with rheumatoid arthritis (RA) or osteoarthritis (OA) were immunostained with antibodies to endocan and vascular endothelial growth factor (VEGF). Subsequently, synovial cells and human umbilical vein endothelial cells were cultured and stimulated with interleukin-1 $\beta$ (IL-1 $\beta$ ) or adiponectin. The mRNA and protein levels of endocan were evaluated by polymerase chain reaction and ELISA, respectively. Endocan expression was markedly increased in the inflammatory sites of RA synovial tissues. In OA tissues, endocan expression was higher in tissues displaying moderate and severe inflammation than in those
\end{abstract}

Correspondence to: Professor Kyoung Soo Kim, East-West Bone and Joint Research Institute, Kyung Hee University Hospital at Gangdong, Kyung Hee University, 149 Sangil-dong, Gangdong-gu, Seoul 134-727, Republic of Korea

E-mail: labrea46@naver.com

Dr Hyung-In Yang, Division of Rheumatology, Department of Internal Medicine, Kyung Hee University Hospital at Gangdong, Kyung Hee University Medical School, 149 Sangil-dong, Gangdong-gu, Seoul 134-727, Republic of Korea

E-mail: yhira@khu.ac.kr

Abbreviations: HUVEC, human umbilical vascular endothelial cells; RA, rheumatoid arthritis; ESM-1, endothelial cell-specific molecule; FLSs, fibroblast-like synoviocytes; siRNA, small interfering RNA

Key words: endocan, endothelial cell-specific molecule, adiponectin, rheumatoid arthritis, angiogenesis with mild inflammation. In vitro expression levels of endocan and VEGF in endothelial and synovial cells were differentially increased in response to IL-1 $\beta$ stimulation. Adiponectin was a more potent stimulant of endocan than IL-1 $\beta$ at their respective physiological concentrations in synovial cells. Endocan silencing by small interfering RNA transfection of synovial cells decreased in vitro cell migration and invasion. In conclusion, adiponectin is an important factor in the stimulation of endocan expression in synovial cells. Adiponectin-induced endocan expression in synovial cells may stimulate cell migration and invasion as well as angiogenesis in the pannus of arthritic joints.

\section{Introduction}

Rheumatoid arthritis (RA) is a systemic disease characterized by synovial inflammation. The combination of the proliferation of synovial lining cells and the infiltration of inflammatory cells, including monocytes and activated leukocytes, into joint tissues contributes to 'pannus' tissue formation, tumor-like growth and eventually to extensive synovial inflammation and joint destruction $(1,2)$. Thus, the continued neovascularization of pannus tissues may facilitate the penetration of inflammatory cells into the synovium and thereby stimulate pannus formation (3). Increased angiogenesis is one characteristic of RA, and rheumatoid joints also contain elevated levels of pro-angiogenic molecules, including vascular endothelial growth factor (VEGF), basic fibroblast growth factors (FGF), hypoxia-inducible factor-1, and angiopoietins (4). Furthermore, preclinical studies have indicated that angiogenesis inhibitors are able to reduce pannus formation, inflammation and joint erosion; and that therapeutic targeting of angiogenesis has demonstrated beneficial effects in the treatment of diseases, including colorectal, kidney and lung cancer $(4,5)$. Thus, angiogenesis inhibitors may be developed as potential therapies.

Endocan, which was previously named endothelial cell-specific molecule (ESM-1), has been studied as a pro-angiogenic factor in tumor tissues (6). It was 
originally cloned from human endothelial cells in 1996 by Lassalle et al (7). Structurally, endocan is a $50 \mathrm{kDa}$ secretory proteoglycan composed of a mature polypeptide of 165 amino acids with a single dermatan sulfate chain covalently linked to $\operatorname{Ser}^{137}$ (6). It is a relatively unusual molecule in that it is able to freely circulate in the blood and carries only one GAG chain. Endocan binds CD11a/CD18 integrin (also known as leukocyte function-associated antigen-1) on human leukocytes, inhibiting its binding to intercellular adhesion molecule 1 and thereby preventing transendothelial migration (7-9). The silencing of endocan in hepatocellular carcinoma resulted in decreased cell migration, invasion and survival (10). Furthermore, endocan has been suggested to be a specific biomarker of tip cells during neoangiogenesis (6). The expression of endocan is upregulated by pro-inflammatory molecules, including tumor necrosis factor alpha (TNF- $\alpha$ ), as well as pro-angiogenic molecules, including VEGF and FGF-2 $(6,11,12)$.

These physiological functions of endocan may additionally be involved in the angiogenesis of pannus in rheumatoid arthritic joints, which are characterized by tumor-like growth. To the best of our knowledge, the relevance of endocan to RA has not previously been studied. In addition, a previous study by our group indicated that adiponectin, which was recently demonstrated to be involved in RA pathogenesis, stimulated the expression of VEGF in FLSs to the same extent as interleukin-1 $\beta$ (IL-1 $\beta$ ), one of the most important stimulators of FLSs. This result suggested that adiponectin was also an important stimulant of angiogenesis in arthritic joints (13). In the present study, endocan expression in arthritic joints was evaluated and those cells which contributed most to endocan production were identified. Furthermore, the effects of adiponectin on the expression of endocan in the FLSs of arthritic joints were examined. In the present study, for the first time, to the best of our knowledge, it is concluded that endocan expression is increased in arthritic synovial tissues and that adiponectin is an important factor involved in mediating the increased endocan expression observed in synovial cells.

\section{Materials and methods}

Synovial tissues and joint fluid collection. Synovial tissues were collected from patients with RA or OA following the attainment of informed consent. Patients met the 1987 American College of Rheumatology criteria for the diagnosis of RA, had been treated with non-biological, disease-modifying anti-rheumatic drugs and had undergone therapeutic joint surgery. Ethical approval was obtained from the Institutional Review Board for Human Research of Kyung Hee University Hospital at Gangdong, Republic of Korea.

Cell culture. FLSs from patients with RA were isolated and grown in Dulbecco's modified Eagle's medium (DMEM, low glucose; Gibco Invitrogen Inc., Grand Island, NY, USA) as described previously (13). Human umbilical vein endothelial cells were obtained from Cell Applications Inc. (San Diego, CA, USA). Once the cells had grown to confluence, they were split at a 1:4 ratio. Passages three to six were used for all experiments. The cells were treated with IL-1 $\beta$ (0.1 or $1 \mathrm{ng} / \mathrm{ml}$; ProSpec, Rehovot, Israel) or adiponectin (1 or $10 \mu \mathrm{g} / \mathrm{ml}$; ProSpec). Culture supernatants were collected for the analysis of VEGF and endocan by ELISA, and the cells were used for total RNA extraction.

Measurement of gene expression by ELISA. The cells $\left(2.5 \times 10^{5}\right.$ cells $/ 60 \mathrm{~mm}$ dish $/ 2 \mathrm{ml}$ serum-free media) were treated with recombinant adiponectin (1 or $10 \mu \mathrm{g} / \mathrm{ml})$ or IL-1 $\beta$ (0.1 or $1 \mathrm{ng} / \mathrm{ml}$; ProSpec, Rehovot, Israel). Conditioned media was collected following $24 \mathrm{~h}$. Briefly, culture supernatants were centrifuged and the supernatants were collected and analyzed for endocan (Lunginnov, Lille, France) and VEGF (R\&D Systems Inc., Minneapolis, MN, USA) with ELISA kits. Three independent experiments were performed in quadruplicate.

Polymerase chain reaction (PCR) analysis of messenger RNA ( $m R N A$ ) expression levels. Culture supernatants were harvested and the cells were subsequently used to measure gene expression levels, as described previously (14). Briefly, complementary DNA was synthesized from $1 \mu \mathrm{g}$ of total RNA in a $20 \mu 1$ reverse transcription reaction mixture. For semi-quantitative PCR, aliquots of cDNA were amplified with the primers in a $25-\mu 1$ PCR mixture containing $1 \mathrm{X}$ PCR buffer, 0.625 units TaKaRa Ex TaqTM HS and $0.2 \mu \mathrm{M}$ specific upstream primers, according to the manufacturer's instructions (TaKaRa Bio, Kyoto, Japan). The PCR conditions for VEGF and endocan were as follows: $95^{\circ} \mathrm{C}$ for $45 \mathrm{sec}, 55-60^{\circ} \mathrm{C}$ for $45 \mathrm{sec}$ and $72^{\circ} \mathrm{C}$ for $45 \mathrm{sec}$; repeated for $30-33$ cycles. The PCR products were subjected to electrophoresis on a $1.5 \%$ agarose gel containing ethidium bromide (Bio-Rad, Hercules, CA, USA) and the bands were visualized under ultraviolet light. For quantitative PCR, the reaction was performed using the LightCycler PCR system (Roche Diagnostics, Meylan, France) and the DNA binding SYBR Green I dye was used to detect the PCR products. Product specificity was determined by melting curve analysis as described in the LightCycler manual. Results are expressed as ratios of endocan transcripts to $\beta$-actin transcripts, with the quantity of transcripts in each sample expressed as a copy number. The primers were synthesized by Bioneer Co. Ltd. (Seoul, Korea), and their sequences are listed in Table I.

Transfection of small interfering RNA (siRNA). FLSs were transiently transfected with siRNA that targeted endocan (Bioneer Co. Ltd.) in Lipofectamine 2000 (Gibco), according to the manufacturer's instructions. Briefly, siRNA $(1 \mu \mathrm{g})$ for endocan (GenBank accession number NM_007036.3) was suspended in $100 \mu 1$ Lipofectamine solution and mixed with an equal volume of serum-free DMEM (Gibco). The mixture was added to $5 \times 10^{5}$ FLSs cultured in $100-\mathrm{mm}$ dishes. (BD Biosciences, Franklin Lakes, NJ, USA) Control siRNA was used as a negative control. Following $6 \mathrm{~h}$ of incubation, the transfected cells were washed twice with phosphate-buffered saline, replenished with fresh medium and grown under IL-1 $\beta$ stimulation $(10 \mathrm{ng} / \mathrm{ml})$ for $24 \mathrm{~h}$. The knockdown of endocan was determined by reverse transcription PCR. Three independent experiments using the synovial cells of one patient with RA were performed in quadruplicate.

Cell migration and invasion assays. Migration and invasion were examined by Transwell assay using a CytoSelectTM 24-Well kit (Cell Biolabs, Inc., San Diego, CA, USA), according 
Table I. The sequence of polymerase chain reaction primers used in this experiment.

\begin{tabular}{ll}
\hline Primer name & \multicolumn{1}{c}{ Primer sequence } \\
\hline MMP-1 sense & 5'-CCT AGC TAC ACC TTC AGT GG-3' \\
MMP-1 antisense & 5'-GCC CAG TAC TTA TTC CCT TT-3' \\
MMP-13 sense & 5'-TTG AGG ATA CAG GCA AGA CT-3' \\
MMP-13 antisense & 5'-TGG AAG TAT TAC CCC AAA TG-3' \\
MMP-2 sense & 5'-ACT TCA GGC TCT TCT CCT TT-3' \\
MMP-2 antisense & 5'-TTC AGA CAA CCT GAG TCC TT-3' \\
Endocan sense & 5'-TGC CTG AAA TTC CCC TTC TT-3' \\
Endocan antisense & 5'-TTC CTC ATT ACG GGA GAC CC-3' \\
$\beta$-actin sense & 5'-TCA TGA GGT AGT CAG TCA GG-3' \\
$\beta$-actin antisense & 5'-CTT CTA CAA TGA GCT GCG TG-3' \\
\hline
\end{tabular}

MMP, matrix metalloproteinase.

to the manufacturer's instructions. For the migration assay, briefly, the inner chambers of the transwells containing polycarbonate membrane inserts were seeded with $0.3 \mathrm{ml}$ synovial cells $\left(0.6 \times 10^{5}\right.$ cells/well $)$ that were transfected with endocan siRNA or control siRNA. Media containing $10 \%$ fetal bovine serum (Sigma-Aldrich St. Louis, MO, USA) was added to the lower well of the migration plate. IL- $1 \beta$ was added to the upper well containing the cells which were activated with IL-1 $\beta$ for $24 \mathrm{~h}$. The migrated cells were stained with a cell staining solution and extracted with an extraction solution (both Cell Biolabs, San Diego, CA, USA) according to the manufacturer's instructions. The optical density of the extracted solution was measured at $560 \mathrm{~nm}$ using an Emax Microplate Reader (Molecular Devices, Sunnyvale, CA, USA). For the invasion assay, the kit required a 24-well plate containing polycarbonate membrane inserts; the upper surface of the insert membrane was coated with a uniform layer of dried basement membrane matrix solution. This basement membrane layer served as a barrier to discriminate invasive from non-invasive cells. The invasion assay was performed simultaneously using an identical protocol to that used for the migration assay but with a different insert.

Histopathology. Specimens were fixed in $10 \%$ buffered formalin (DNA Korea, Incheon, Korea), processed routinely and embedded in paraffin. Sections $(4 \mu \mathrm{m})$ of paraffin blocks were cut and subsequently stained with hematoxylin and eosin (H\&E) and immunohistochemical stain. Immunohistochemical staining was performed in a Bond-Max automated slide stainer (Leica Microsystems, Newcastle, UK) using monoclonal mouse endocan/ESM-1 antibody $(1 / 5,000$; LIA-0901, Lunginnov, Lille, France). Antigens (Leica Microsystems) were retrieved with epitope retrieval solution 1 (Leica Microsystems). Slides were incubated with the antibody at room temperature for $20 \mathrm{~min}$ and subsequently incubated with a biotinylated secondary antibody for $8 \mathrm{~min}$. The resulting complexes were detected using avidin-peroxidase conjugate polymer. Color was developed using 3,3'-diaminobenzidine (ScyTek, Logan, UT, USA). Mayer's hematoxylin (Leica Microsystems) was used as a counterstain. Positive and negative control staining were used. For the evaluation of endocan and VEGF expression, the area of cytoplasmic staining was determined as a percentage and scored as follows: 1, staining in $<10 \%$ of cells; 2 , staining in $10-50 \%$ of cells and 3 , staining in $>50 \%$ of cells.

Statistical analysis. The in vitro experimental data were expressed as the mean \pm standard error of the mean (SEM) of quadruplicate samples. Differences between groups were assessed using repeated analysis of variance followed by the Dunnett multiple comparison test. The degree of inflammation observed in H\&E-stained sections, mRNA expression levels determined by PCR and the cell migration and invasion tests in endocan siRNA-transfected cells were compared between groups with the Mann-Whitney U test. Prism software 5.02 (GraphPad Software, Inc., San Diego, CA, USA) was used for statistical analysis and graphing. $\mathrm{P}<0.05$ was considered to indicate a statistically significant difference between values.

\section{Results}

Increased expression of endocan and VEGF in inflammatory arthritic joints. Synovial tissues from patients with RA (n=3) and OA $(n=10)$ were immunohistochemically stained with antibodies to endocan and VEGF in order to examine their expression in arthritic joints. Endocan expression was detected in vascular endothelial cells, infiltrated lymphocytes and proliferating synovial cells in synovial tissues of RA (Fig. 1A). Based on synovial tissues from three RA patients, expression of endocan in synovial cells was correlated with the degree of inflammation, though its expression remained unaltered in vessels regardless of the degree of inflammation. Similarly, VEGF expression was also detected in these cells; however, endocan expression in RA synovial cells was markedly higher than that of VEGF. Subsequently, to evaluate endocan and VEGF expression in synovial tissues of OA patients and determine their association with the degree of inflammation, OA synovial tissues $(n=10)$ were divided into mild, moderate, and severe groups according to the degree of inflammation observed. The degree of inflammation was evaluated by the number of infiltrated immune cells, based on a relative degree 
A

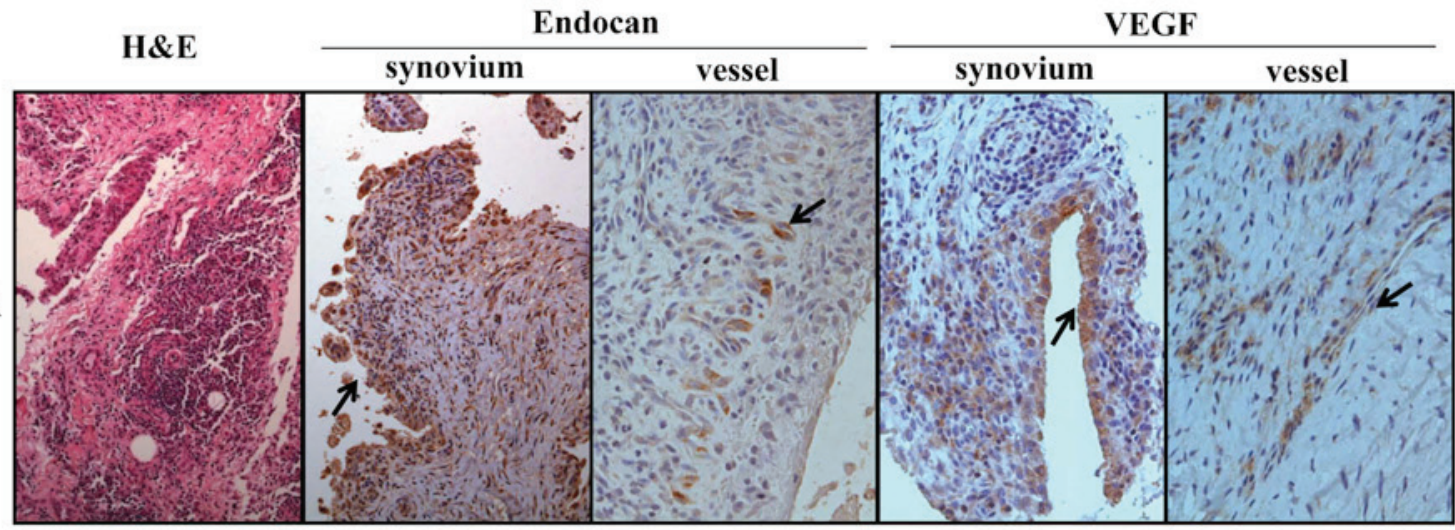

B
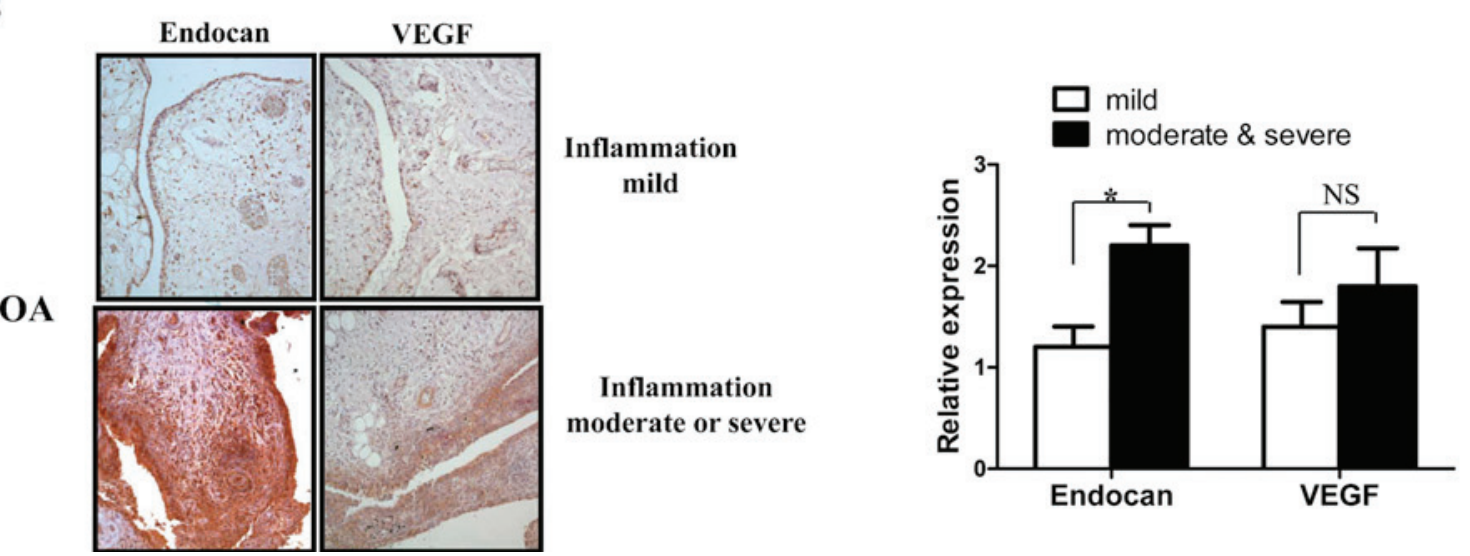

Figure 1. Expression of endocan and VEGF in synovial tissues of joints with RA or OA. (A) Expression of endocan and VEGF in arthritic tissues of RA patients $(n=3)$ was evaluated by immunohistochemistry. The arrows indicate significant expression of endocan or VEGF in the synovium and vessels. (B) Expression levels of endocan and VEGF were compared in the arthritic tissues of OA patients $(n=10)$, which were divided into three groups depending on the degree of inflammation; mild, moderate or severe. On the graph, the expression values of endocan and VEGF in OA arthritic tissues are expressed as the mean \pm standard error of the mean. * $\mathrm{P}<0.05$; NS, not significant. VEGF, vascular endothelial growth factor; RA, rheumatoid arthritis; OA, osteoarthritis; H\&E, hematoxylin and eosin.

of inflammation. The endocan expression levels in OA tissues with moderate and severe inflammation were approximately two-fold higher than those of tissues with mild inflammation. Similarly, VEGF expression was higher in tissues exhibiting moderate and severe inflammation; however, this had no statistical significance (Fig. 1B). These results suggested that endocan expression was significantly upregulated in inflamed arthritic synovial tissues.

Comparison of endocan and VEGF expression in endothelial cells and FLSs under pro-inflammatory stimulation. To further provide indirect evidence of the expression patterns of endocan and VEGF in arthritic tissues, two gene expression patterns in cultured endothelial cells and FLSs under pro-inflammatory stimulation were investigated in vitro by PCR. The endocan gene was constitutively expressed in cultured endothelial cells, and its expression was not significantly increased in response to IL-1 $\beta$ (0.1-1 ng/ml) (Fig. 2A). By contrast, VEGF expression was significantly increased following IL-1 $\beta$ stimulation in endothelial and synovial cells. Consistent with the observed RNA expression levels, endocan protein was constitutively expressed in cultured endothelial cells and its expression levels (mean \pm SEM) were not increased in response to stimulation with $1 \mathrm{ng} / \mathrm{ml}$ IL-1 $\beta(67.44 \pm 3.31 \mathrm{ng} / \mathrm{ml}$ vs. $54.63 \pm 2.75 \mathrm{ng} / \mathrm{ml})$ (Fig. 2B).
However, the protein expression levels of endocan in synovial cells in response to IL-1 $\beta(1 \mathrm{ng} / \mathrm{ml})$ were increased $\sim 5$-fold compared to those with no stimulation $(0.58 \pm 0.04$ vs. $0.12 \pm 0.01 \mathrm{ng} / \mathrm{ml})$. The relative protein expression levels of endocan in endothelial cells were $\sim 100$-fold greater than those of FLSs $(54.63 \pm 2.75$ vs. $0.58 \pm 0.04 \mathrm{ng} / \mathrm{ml})$; however, IL-1 $\beta$ significantly increased the expression of endocan in synovial cells (Fig. 2B). Conversely, the relative VEGF protein expression levels in synovial cells were $\sim 30$-fold higher than those in endothelial cells $(89.94 \pm 3.29$ vs. $3.12 \pm 0.34 \mathrm{pg} / \mathrm{ml})$, although IL-1 $\beta$ stimulation induced VEGF expression in both cell types. These results suggested that endocan was mainly produced in endothelial cells and partly produced in synovial cells of arthritic joints under inflammatory stimulation, whereas VEGF was produced equally in both cell types.

Effect of adiponectin on the expression of endocan in synovial cells. In previous studies by our group, adiponectin was shown to have an important function in angiogenesis, as it was a potent stimulant of VEGF in synovial cells, similarly to IL-1 $\beta$ (13). Therefore, the stimulatory effect of adiponectin on endocan expression in arthritic joints was investigated. FLSs and endothelial cells were stimulated with adiponectin or IL-1 $\beta$ (Fig. 3). The expression pattern of endocan was compared in three different cell types. Endocan expression 
A
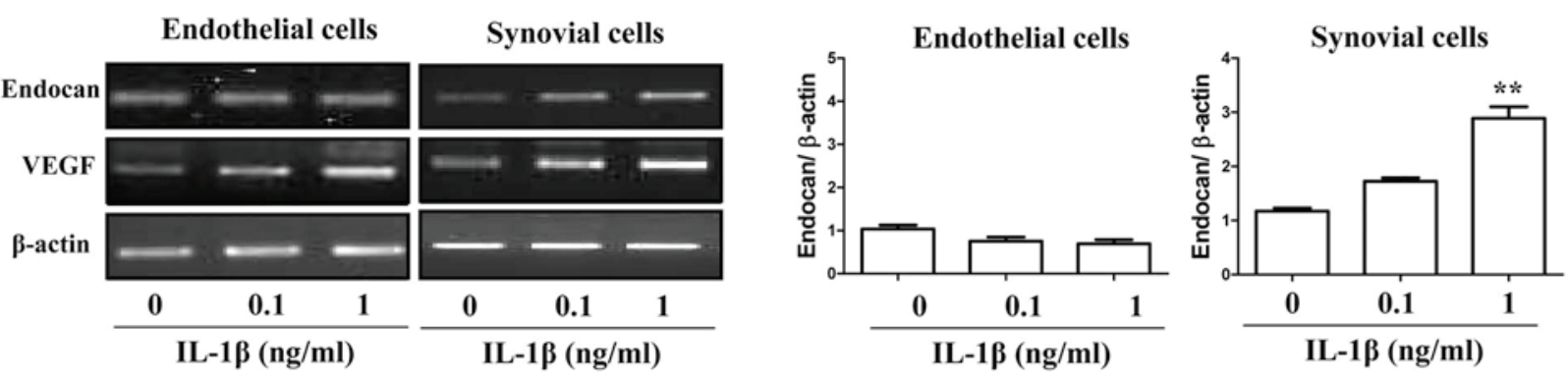

B
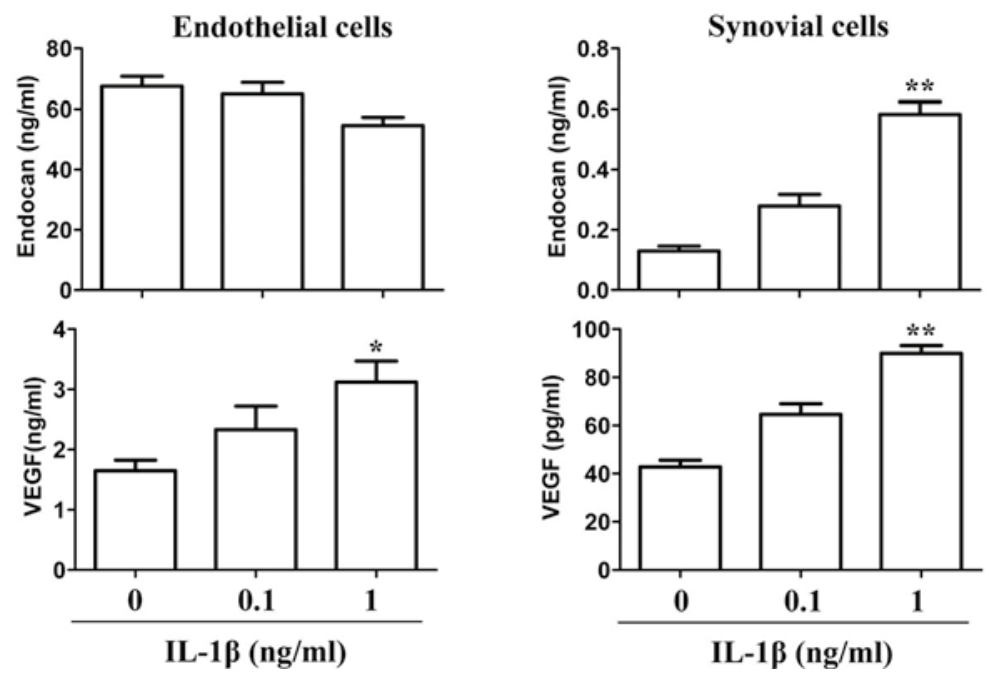

Figure 2. Comparative expression of endocan and VEGF in endothelial cells and fibroblast-like synoviocytes under pro-inflammatory stimulation. Endothelial cells and synovial cells were stimulated with IL-1 $\beta(0.1$ or $1 \mathrm{ng} / \mathrm{ml})$. Levels of endocan and VEGF were evaluated by (A) semi-quantitative polymerase chain reaction and (B) ELISA. The data are representative of three independent experiments, each performed in quadruplicate. Values are expressed as the mean \pm standard error of the mean. "P<0.05; ${ }^{* *} \mathrm{P}<0.01$ compared with untreated cells. VEGF, vascular endothelial growth factor; IL- $1 \beta$, interleukin-1 $\beta$.

A

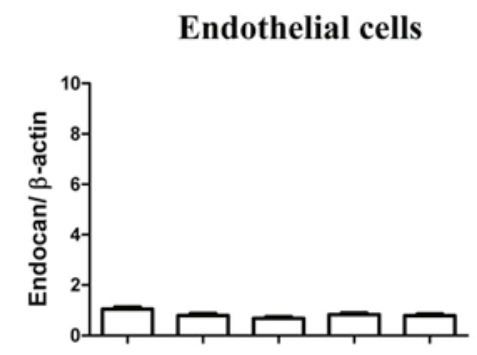

B

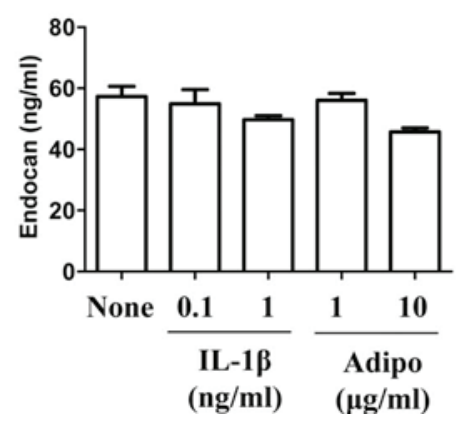

Osteoblast
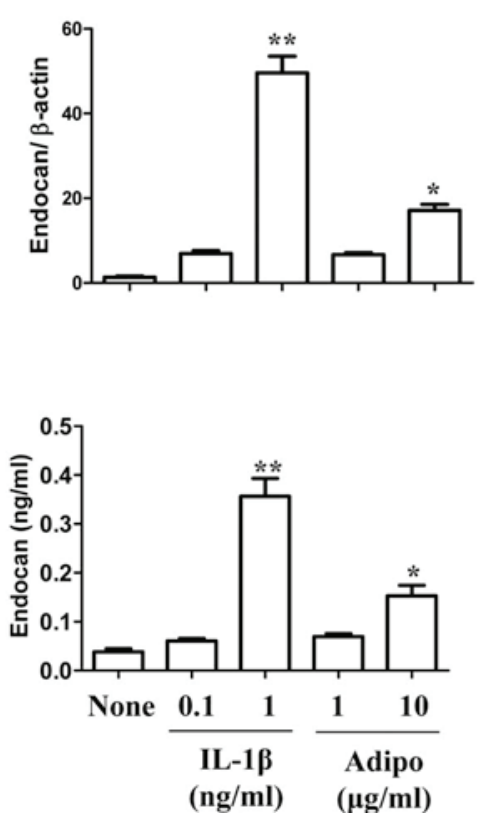

Synovial cells
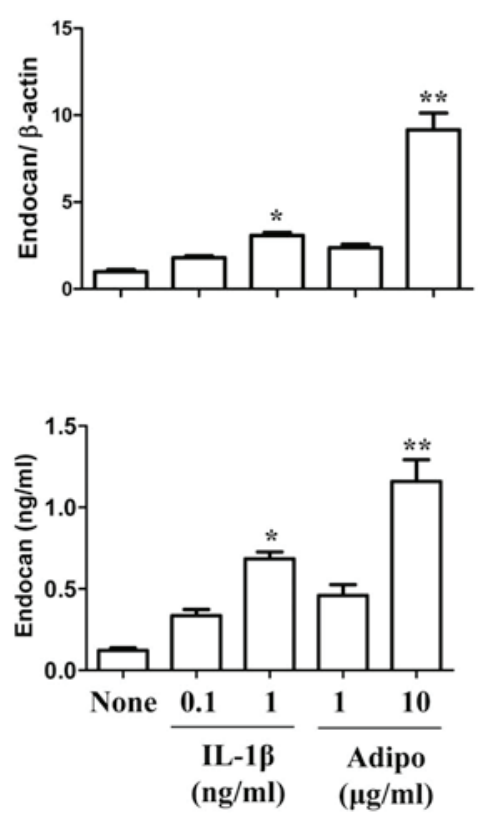

Figure 3. Effect of adiponectin on endocan expression in synovial cells. Endothelial and synovial cells were stimulated with IL-1 $\beta$ (0.1 or $1 \mathrm{ng} / \mathrm{ml})$ or adiponectin $(1$ or $10 \mu \mathrm{g} / \mathrm{ml})$. Expression levels of endocan were evaluated by (A) polymerase chain reaction and (B) ELISA. Data shown are representative of three independent experiments, each performed in quadruplicate. ${ }^{*} \mathrm{P}<0.05 ;{ }^{* * *} \mathrm{P}<0.01$ compared with untreated cells. IL-1 $\beta$, interleukin-1 $\beta$; adipo, adiponectin. 


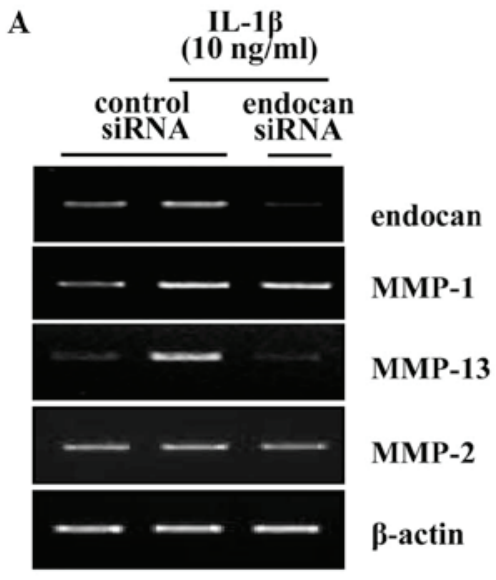

B
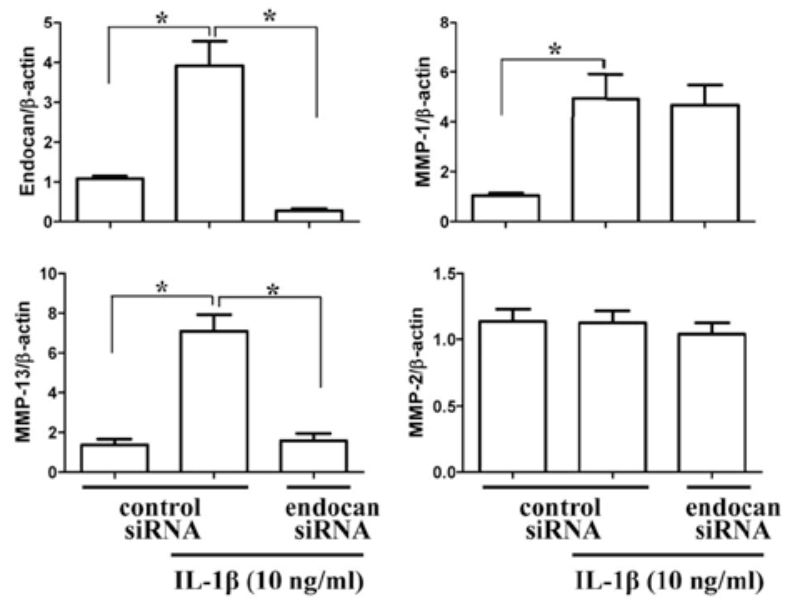
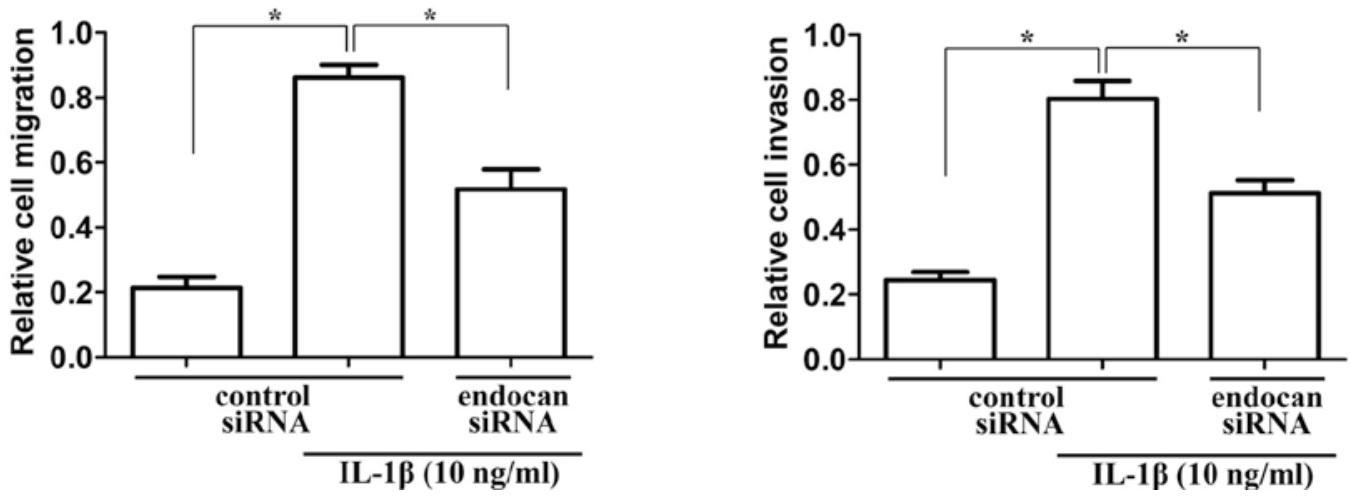

Figure 4. Role of the endocan gene on the invasion and migration of synovial cells from a patient with rheumatoid arthritis. (A) Levels of MMP expression determined by reverse transcription polymerase chain reaction in endocan gene-silenced synovial cells through endocan siRNA transfection. (B) Cell migration and invasion assay in endocan gene-silenced synovial cells. Synovial cells were transfected with control or endocan siRNA and stimulated with IL-1 $\beta$ $(10 \mathrm{ng} / \mathrm{ml})$. Data are representative of three independent experiments. Values are expressed as the mean \pm standard error of the mean. ${ }^{*} \mathrm{P}<0.05$. siRNA, small interfering RNA; IL-1 $\beta$, interleukin-1 $\beta$; MMP, matrix metalloproteinase.

in adiponectin $(10 \mu \mathrm{g} / \mathrm{ml})$-stimulated synovial cells was $\sim 4$-fold and $\sim 3$-fold higher than in IL-1 $\beta$-stimulated cells at concentrations of 0.1 and $1 \mathrm{ng} / \mathrm{ml}$, respectively. Considering the physiological concentrations of IL- $1 \beta(<0.1 \mathrm{ng} / \mathrm{ml})$ and adiponectin (1-10 $\mu \mathrm{g} / \mathrm{ml})$ in joint fluids, adiponectin is more likely to be significantly involved in the production of endocan in synovial cells. However, endocan expression was not stimulated by adiponectin in endothelial cells. In addition, the expression of VEGF and endocan in osteoblasts was stimulated by adiponectin in a similar pattern to that of synovial cells (data not shown). These results suggested that endocan was constitutively expressed in endothelial cells, and that adiponectin was a more potent stimulant of endocan production in synovial cells and osteobalsts than IL-1 $\beta$ at physiological concentrations.

Endocan gene knockdown by siRNA inhibits the invasiveness and migration of synovial cells. To provide insight into the role of the endocan gene in synovial cell migration and invasion in arthritic joints, the gene was silenced in vitro by siRNA transfection. Matrix metalloproteinase (MMP) genes, which are associated with invasion and migration, were analyzed for mRNA expression levels in endocan gene-silenced synovial cells (Fig. 4A). Gene levels of collagenase (MMP-1 and MMP-13) and gelatinase (MMP-2) were investigated by RT-PCR. As indicated in Fig. 4A, mRNA levels of MMP-1 and MMP-2 were not significantly altered, but MMP-13 levels were significantly decreased in cells transfected with endocan siRNA and treated with IL-1 $\beta(10 \mathrm{ng} / \mathrm{ml})$ for $24 \mathrm{~h}$. Subsequently, the effects of endocan gene knockout on the invasion and migration of FLSs were investigated. As demonstrated in Fig. 4B, endocan gene silencing significantly decreased cell migration and invasion of FLSs under inflammatory conditions. These results suggested that the endocan gene may have an important role in FLSs in mediating the pannus invasion of cartilage and bone in arthritic joints.

\section{Discussion}

In the current study, we hypothesized that the previously reported physiological roles of endocan in tumors were involved in the pathogenesis and progression of RA. In particular, endocan was reported to be involved in angiogenesis and tumor invasion $(15,16)$. RA is characterized by excessive angiogenesis, which may be essential in the pathogenesis of the disease (17). Furthermore, the pannus tissue in RA joints exhibits aggressive, tumor-like growth and invades and erodes the surrounding cartilage and subchondral bones (18). Thus, 
the present study investigated whether endocan expression was increased in arthritic tissues. Immunohistochemistry revealed that its expression was increased in severe inflammatory arthritic tissues. The increased expression of endocan in arthritic joints may be responsible for excessive angiogenesis and pannus invasion as well as in the recruitment of circulating lymphocytes to inflammatory sites and leukocyte adhesion and activation (9).

Subsequently, which cells in arthritic joints are mainly responsible for the production of endocan and how they are regulated by inflammatory stimuli was examined. The results suggested that endocan was mainly produced in the endothelial cells of arthritic joints, even following inflammatory stimulation. Endocan was previously reported to be preferentially expressed in the tumor endothelium in vivo, which supported this result (19). VEGF is an important factor that stimulates endocan expression in the endothelium (15). Furthermore, endocan secretion was significantly increased in response to TNF- $\alpha$, and the spontaneous and TNF- $\alpha$-induced secretion of endocan-1 was inhibited by interferon- $\gamma$ (20). However, the results of the present study indicated that endocan expression was not significantly increased in response to IL-1 $\beta$ ( $1 \mathrm{ng} / \mathrm{ml})$. In addition, endocan expression was elevated in bronchial and renal epithelia (20). Adipocytes, which actively produce endocan (21), also increased their endocan expression in response to phorbol ester, an activator of protein kinase C, and retinoic acid (22). In the present study, synovial cells significantly increased the expression of endocan in response to inflammatory stimuli, including IL-1 $\beta$. Meanwhile, adiponectin, an adipokine, was involved in the pathogenesis and progression of arthritis in joints. Thus, the role of adiponectin in the stimulation of endocan expression in arthritic joints was studied. To the best of our knowledge, the present study was the first to describe the effect of adiponectin on the expression of endocan.

To evaluate the role of endocan in arthritic joints, the endocan gene was knocked down by endocan siRNA in FLSs. Consistent with previous results in other cell types (10), endocan silencing in FLSs decreased the levels of cell migration and invasion in in vitro assays. The results of the present study indicated that the decrease in cell migration and invasion may be associated with downregulation of the MMP-13 gene caused by endocan silencing, similar to results observed in a previous study (23).

In conclusion, to the best of our knowledge, the present study indicated, for the first time, that endocan expression was detected in arthritic joint tissues and that the expression was higher at severe inflammatory sites than at mild inflammatory sites. Therefore, endocan may be involved in synovial cell migration and invasion of pannus tissue in arthritic joints. The expression regulation of endocan and its major sources in arthritic joints remain to be further investigated. Furthermore, in order to present a potential therapeutic target against rheumatoid arthritis, the in vivo role of endocan should be investigated in animal models of arthritis through knockout of the endocan gene.

\section{Acknowledgements}

The present study was supported by the Basic Science Research Program through the National Research Foundation of Korea and funded by the Ministry of Education, Science and Technology (Korea; grant nos. 2011-0009061 and 2010-0024089).

\section{References}

1. Gravallese EM and Monach PA: Rheumatoid synovitis and pannus. In: Rheumatology. Hochberg MC, Silman AJ, Smolen JS Weinblatt ME and Weisman MH (eds). 4th edition. Elsevier Ltd, London, UK, pp841-865, 2008.

2. Bresnihan B: Pathogenesis of joint damage in rheumatoid arthritis. J Rheumatol 26: 717-719, 1999

3. Szekanecz Z and Koch AE: Mechanisms of disease: angiogenesis in inflammatory diseases. Nat Clin Pract Rheumatol 3: 635-643, 2007.

4. Schoettler N and Brahn E: Angiogenesis inhibitors for the treatment of chronic autoimmune inflammatory arthritis. Curr Opin Investig Drugs 10: 425-433, 2009.

5. Lainer-Carr D, and Brahn E: Angiogenesis inhibition as a therapeutic approach for inflammatory synovitis. Nat Clin Pract Rheumatol 3: 434-442, 2007.

6. Sarrazin S, Adam E, Lyon M, Depontieu F, Motte V, Landolfi C, Lortat-Jacob H, Bechard D, Lassalle P and Delehedde M: Endocan or endothelial cell specific molecule-1 (ESM-1): a potential novel endothelial cell marker and a new target for cancer therapy. Biochim Biophys Acta 1765: 25-37, 2006.

7. Lassalle P, Molet S, Janin A, Heyden JV, Tavernier J, Fiers W, Devos R and Tonnel AB: ESM-1 is a novel human endothelial cell-specific molecule expressed in lung and regulated by cytokines. J Biol Chem 271: 20458-20464, 1996.

8. Béchard D, Gentina T, Delehedde M, Scherpereel A, Lyon M, Aumercier M, Vazeux R, Richet C, Degand P, Jude B, et al: Endocan is a novel chondroitin sulfate/dermatan sulfate proteoglycan that promotes hepatocyte growth factor/scatter factor mitogenic activity. J Biol Chem 276: 48341-48349, 2001.

9. Béchard D, Scherpereel A, Hammad H, Gentina T, Tsicopoulos A, Aumercier M, Pestel J, Dessaint JP, Tonnel AB and Lassalle P: Human endothelial-cell specific molecule-1 binds directly to the integrin CD11a/CD18 (LFA-1) and blocks binding to intercellular adhesion molecule-1. J Immunol 167: 3099-3106, 2001.

10. Kang YH, Ji NY, Lee CI, Lee HG, Kim JW, Yeom YI, Kim DG, Yoon SK, Kim JW, Park PJ and Song EY: ESM-1 silencing decreased cell survival, migration, and invasion and modulated cell cycle progression in hepatocellular carcinoma. Amino Acids 40: 1003-1013, 2011.

11. Maurage CA, Adam E, Minéo JF, Sarrazin S, Debunne M, Siminski RM, Baroncini M, Lassalle P, Blond S and Delehedde M: Endocan expression and localization in human glioblastomas. J Neuropathol Exp Neurol 68: 633-641, 2009.

12. Grigoriu BD, Depontieu F, Scherpereel A, Gourcerol D, Devos P, Ouatas T, Lafitte JJ, Copin MC, Tonnel AB and Lassalle P: Endocan expression and relationship with survival in human non-small cell lung cancer. Clin Cancer Res 12: 4575-4582, 2006.

13. Choi HM, Lee YA, Lee SH, Hong SJ, Hahm DH, Choi SY, Yang HI, Yoo MC and Kim KS: Adiponectin may contribute to synovitis and joint destruction in rheumatoid arthritis by stimulating vascular endothelial growth factor, matrix metalloproteinase-1, and matrix metalloproteinase-13 expression in fibroblast-like synoviocytes more than proinflammatory mediators. Arthritis Res Ther 11: R161, 2009.

14. Kim KS, Park EK, Ju SM, Jung HS, Bang JS, Kim C, Lee YA, Hong SJ, Lee SH, Yang HI and Yoo MC: Taurine chloramine differentially inhibits matrix metalloproteinase 1 and 13 synthesis in interleukin-lbeta stimulated fibroblast-like synoviocytes. Arthritis Res Ther 9: R80, 2007.

15. Roudnicky F, Poyet C, Wild P, Krampitz S, Negrini F, Huggenberger R, Rogler A, Stöhr R, Hartmann A, Provenzano M, et al: Endocan is upregulated on tumor vessels in invasive bladder cancer where it mediates VEGF-A-induced angiogenesis. Cancer Res 73: 1097-1106, 2013.

16. Aitkenhead M, Wang SJ, Nakatsu MN, Mestas J, Heard C and Hughes CC: Identification of endothelial cell genes expressed in an in vitro model of angiogenesis: induction of ESM-1, (beta) ig-h3, and NrCAM. Microvasc Res 63: 159-171, 2002.

17. Weber AJ and De Bandt M: Angiogenesis: general mechanisms and implications for rheumatoid arthritis. Joint Bone Spine 67: 366-383, 2000.

18. Firestein GS: Evolving concepts of rheumatoid arthritis. Nature 423: 356-361, 2003. 
19. Abid MR, Yi X, Yano K, Shih SC and Aird WC: Vascular endocan is preferentially expressed in tumor endothelium. Microvasc Res 72: 136-145, 2006.

20. Bechard D, Meignin V, Scherpereel A, Oudin S, Kervoaze G, Bertheau P, Janin A, Tonnel A and Lassalle P: Characterization of the secreted form of endothelial-cell-specific molecule 1 by specific monoclonal antibodies. J Vasc Res 37: 417-425, 2000.

21. Janke J, Engeli S, Gorzelniak K, Feldpausch M, Heintze U, Böhnke J, Wellner M, Herse F, Lassalle P, Luft FC and Sharma AM: Adipose tissue and circulating endothelial cel specific molecule-1 in human obesity. Horm Metab Res 38 : 28-33, 2006.
22. Wellner M, Herse F, Janke J, Gorzelniak K, Engeli S, Bechart D, Lasalle P, Luft FC and Sharma AM: Endothelial cell specific molecule-1 - a newly identified protein in adipocytes. Horm Metab Res 35: 217-221, 2003

23. Kang YH, Ji NY, Han SR, Lee CI, Kim JW, Yeom YI, Kim YH, Chun HK, Kim JW, Chung JW, et al: ESM-1 regulates cell growth and metastatic process through activation of NF- $\kappa \mathrm{B}$ in colorectal cancer. Cell Signal 24: 1940-1949, 2012. 